\title{
Mobile Cloud-based Big Data Library Management System
}

\author{
Jing Li and Chunying Cui \\ Institute of Computer Science and Technology, ZhouKou Normal University, \\ Zhoukou,Henan ,466001, China \\ zknulj@163.com
}

\begin{abstract}
Mobile Internet can enable the reader to obtain a digital library resources from anywhere, but because of the huge amount of data in large data quickly retrieve the required information, the problem faced by mobile cloud library, aiming at this problem, we propose a big data mobile cloud Books and memory retrieval system with associated services, according to this system, it is possible to quickly search and develop reading programs for the reader's query and search time.
\end{abstract}

Keywords: Mobile cloud computing; Library management; Random matrix

\section{Introduction}

Mobile Internet-based book reading mode, with the rise of the fifth generation of mobile communications, has become the main mode of reading; As the Internet world will be able to every corner of the reading material, especially books displayed on mobile phones and other portable electronic products, so a huge amount of data, in addition to the amount of data as well as other difficult process huge unexpected features. Data types of big data applications to be processed is very diverse. Data generated from the point of view, might be reading history log output, it may be observations and scientific research units, but also may be user data networking applications. From the storage format, the text may be character format, one that is binary audio, video, possible further compressed encrypted information, and other formats. Data from the organization point of view, it may be structured data in a fixed format, the information may be scattered unstructured data.

Many big data applications need to complete the process within a limited time. Such as information search the web ordering (applications, the need to page hits to update the entire web index weights system, so as to achieve real-time update the order page for the purpose of actual operation, there have been new website on-line and new page hits occurs, the kind used by the application data sets are constantly changing. in order to ensure a better user experience, application timely manner is very important recalculated according to changes in the data set. another example is the weather forecast. this application uses satellite imagery and meteorological data collection, etc., to obtain a large original information. All information gathered by meteorologists to predict the weather models in accordance with the most recent changes in the weather. meteorological information requested in the application of meteorological models need analysis process for a limited time to complete, otherwise you lose the use of weather forecasts is hard to assess the value of big data application data set can provide. larger amount of data does not mean the data sets to produce more practical value. on the one hand, a lot of data collection itself may have some inaccuracies. data collection process is not reliable. characteristics of the reality of things is diversified, the collected data can not accurately describe the full big data application data set may also be contains a lot of redundant information before processing requires a lot of finishing work. on the other hand, large data applications, the data set contains the realization of the law can not be 
assessed, the degree of association between the target data sets and expect to find the unknown, We need to build a different model, at the same time try to set the amount of data.

Dig out useful information from vast amounts of raw data is big data most important work, the information process of exploration and mining requires huge computational support. Now, however, the speed of society has been greater than the data capacity of a computer system to calculate the growth rate. Depicts a comparison of growth rates of big data applications increase the amount of data and computer systems computing power. According to International Data Corporation statistical data now global growth rate has been much higher than the speed of Moore's Law. A large amount of data in the rapidly growing application data to the computer system hardware and software developers has posed a severe challenge. Cloud computing on-demand and pay-per-use IT resource use patterns, reducing the data needed to build a large investment in hardware and software service and maintenance costs, facilitate cost-efficient implementation of large data services. Specifically, cloud computing resources through cloud computing platform for service (laaS infrastructure services, PaaS and SaaS platform services applications and services) in the form of user demand for calls. A variety of commercial cloud services will play through building services for the development of a large portion of data services and provides a luxury extended elastic cloud resource pools. By using the cloud laaS, PaaS or SaaS services, making full use of cloud-demand access, usage-based billing and elastic scalability characteristics, can meet the large demand for data services, the build process for computing or storage resources, and greatly improving big data services development and deployment efficiency. In recent years, with the development of cloud computing technology and matures from a cost and performance on both sides, more and more companies and organizations choose to move applications to the cloud environment in the implementation and development skillfully deploy big data services in the cloud. Discuss how the network of cross-application migration to the cloud, the use of the rich resources in the cloud and cloud-demand calls, pay-per-use advantages of $\mathrm{W}$ to reduce operation and maintenance costs of the application; $\square$ Zhang and others designed a model using optimization in the geographically distributed data resources to efficiently migrate to the cloud, $\mathrm{W}$ efficient processing of large data management and analysis. In addition, the researchers also discussed the Facebook, Twitter and other companies is how to choose to use a private cloud infrastructure to build large data service, and by using MapReduce and Hadoop and other big data technologies and tools to achieve PB-level data processing. At the same time, many scientific computing scientific computing tasks associated with big data services, such as the "Human Genome Project" and "Looking beyond the planet Earth" and other ultra-large-scale data, is deployed on multiple platforms such as cloud Amazon $\square$ EC2, Nimbus and Eucalyptus through collaboration between multiple cloud platform to complete large, complex calculations and data processing tasks ${ }^{[1-3]}$.

In summary, the process of library management tools enabling mobile Internet platform read data is the introduction of mobile cloud computing, can make full use of parallel computing peripherals can achieve rapid retrieval and recommendation, it enables mobile Internet platform library management, artificial Books intelligent recommendation and other functions. For multiple cloud services deployed in large data service, reliability of cloud services combined sister program performance for large data service has a crucial impact. Since the data processing tasks big data services generally have data-intensive or compute-intensive features, if cloud services portfolio to participate in the implementation of the program during the execution of large data service can not provide QoS metrics advertised its value, will result in large data services failed, thereby affecting the user experience. The traditional method uses a combination of QoS metrics to assess the value of the service provider released an evaluation of the combination of this practice in a cloud environment facing $\mathrm{W}$ at issue: a dynamic network environment, the service provider may be commercial speculation and other factors will make QoS value QoS 
values and their actual implementation process service provider released are very different, thereby affecting the credibility of the combination regimen.

The development of cloud storage technology and mature, so QoS history of big data generated during the execution of cloud services maintained. The QoS history record large data cloud services, the service provider using QoS parameters to solve the traditional publishing methods to assess the value of a combination of the implementation of portfolio assessment, the credibility problem facing provides a new idea: the combination of the assessment process QoS values, using cloud services QoS history rather than the service provider issued the combined evaluation of the program. Since the QoS history of big data by the cloud services produced in the course of its objective response service QoS performance in the actual implementation process, the use of cloud services QoS history records is a service combination regimen evaluated, can enhance the collective assessment of credibility, we will find the optimal cloud service discipline combined program approach based QoS history of cloud services, recorded bigger credible portfolio assessment data services. In practice, based on the large data QoS service history discipline credible assessment of co-faced QoS history by a massive big data services brings large computation time consuming problem. Specifically, the assessment is a combination of a typical multi-objective optimization problem, the most direct approach to solve the optimal combination regimen is based on an enumeration of all co-century history QoS scheme, using the average value as a utility function corresponding to the combination of cloud services program the utility value; and then sorted according to the utility value, choose the best service QoS combination regimen involved in mapping and deploy big data services. In a cloud environment, to send such a practice will be faced with huge computational complexity and time-consuming, how to improve the computational efficiency of portfolio assessment is an important issue credible big data services portfolio assessment need to be resolved ${ }^{[4-6]}$. Single cloud service, the QoS indicators include: a service grid, response time, reliability, availability, credibility and so on. Each QoS indicators here mutually different nature, affecting its index value of user satisfaction are also different. Service (QoS) value to affect users are divided into two types: one is the benefit index (Positive type QoS), the higher the value the more the degree of user satisfaction Gao, such as usability, availability and credibility; another indicators are cost index (or called Negative type QoS), the higher the value, the lower the level of user satisfaction.

Data resource layer: data resources layer refers to the distribution of resources in the cloud of data collection, data resources can be provided by each data source provider and to be provided by the network to any user. In a cloud environment, data resources via cloud services package, called user data resources described by matching data speed information resources and services; data resource discovery Layer: Find function data resources layer is based on the needs of large data services from Find data resource layer dispersed throughout the data in the cloud resource provider of the match and meet the application requirements of data resources and the formation of large data resources to be processed through collected as a large input data and services; mission planning layer: when application requirements of large data services too complex, W cause can not be through the use of a single cloud services to meet their compute resources or storage resources requirements, tasks, regulate brake layer is responsible for a large data processing task planning, complex big data split handle computing tasks to a single set of functions and independent sub-tasks, such as storage, offline processing big data and big data online calculation of big data and the like. As the function of the sub-task is relatively simple, single cloud services can meet their computing resources or storage requirements. Further, by between subtasks Collaborating to divide and conquer approach to meet large data services demand for IT resources; credible portfolio assessment layer: Trusted portfolio assessment layer is a mission planning based on large data mission planning layer generated services assess the implementation of cloud services portfolio solutions. 
In general, for large data service mission planning for each sub-task, the number of services to meet the needs of many of its resources, these cloud services with the same or similar functional indicators (eg able to provide on-demand scalable storage or virtual machine resources), but they differ in the non-functional indicator (ie, quality of service QoS) on. Non-functional indicator means that the unit price of cloud services, the failure rate, credit and other indicators, describe the degree of satisfaction of users of the cloud services. Typically, large data applications and services will be given of the leased cloud services QoS constraints. Portfolio assessment is based on QoS constraint index large data service applications demand, combined with each sub-task to meet the needs of cloud services and their QoS parameters, data services builders large selection of QoS optimal combination scheme; due to large data processing having the data size large, complex calculation process characteristics, if any cloud service due to the poor reliability resulting in a large data processing delay or failure, the risk of re-execute large data processing tasks, thus affecting the user experience. Traditional portfolio assessment is the use of QoS data service provider released, the combined evaluation of the program; credible portfolio assessment refers to the process of using execution history QoS services produced in Kyrgyzstan to assess cloud service composition scheme meets big data service QoS demand, and based on this selection QoS optimal portfolio of services solutions for large data processing tasks.

\section{Cloud Computing Theory}

\subsection{Cloud Computing Big Data Development}

Service-Oriented Architecture application as an underlying technology of cloud computing as a service to the data resources, storage resources, computing resources and software application platform resources package, so that the user called resources through a service interface, shielding the resources distributed and heterogeneous impact of resource sharing, cloud computing resources can play all kinds of ubiquitous access, on-demand technology based applications. At the same time, SOA as a service within the field of computing a scalable, loosely coupled services and consumer publishing platform that enables application developers to dynamically integrate or have some combination of services to new cloud services, thereby reducing the difficulty of application development, Suppliers to mention resource utilization, reduce maintenance costs. Data Resources cloud environments generally look built on SOA service model calculations, in order to encapsulate data service resources, data users can obtain data service call Mary over resources. Cloud Environment data resource discovery context, SOA model includes five main entities; data resources service provider, service requestor data resources, data resources and services also registered. Data resources via cloud service providers encapsulate data resources, cloud services in a common interface defines its functional parameters and registration were also registered; service users according to their application needs, service registrations also matching service, select their needs and match service ${ }^{[7-9]}$. Finally, according to the results of matching service, the use of data resources service description node resource information and data resource provider binding, and call the appropriate service resources to obtain the required data.

However, in practical applications, SOA-based data resource management and to find the model, data resources and services registered in the Universal Description, Discovery and Integration protocol is a centralized architecture and periodically synchronize multiple data sources (realization when unified data resource management and search. at 5 environment, large data processing and computing resources rely on data scattered in various data resources distributed nodes in the network, the available data resources and services with a huge and dynamic online or offline feature, so that the data resource management and search methods based registry, faced with a single point of failure, poor scalability, unable to meet the flexible, scalable and robust data service resource discovery 
needs. this is the UDDI protocol is not widely use, SOA centralized service management model gradually fade out and look for reasons based .

Traditional centralized data resource management and search methods can not meet the application requirements of large data service dynamically extensible data resource discovery, researchers for non-centralized data resource management and search, carry out the relevant research work, the most typical the application is the use of P2P technology to manage data resources and $\operatorname{search}^{[10]}$. P2P based data resources and service management to find methods have the advantage of dynamically scalable, single point of failure can be overcome centralized approach in the management of large-scale dynamic resource node faces the problem of poor scalability. In general, P2P network topology is divided into structured and unstructured P2P network P2P network two structures. Wherein the structured $\mathrm{P} 2 \mathrm{P}$ network is a connection between extension structure composed of full node P2P network formed by a mesh, ring, or torus butterfly like. Friends these topologies are generally constructed by distributed hash table. Join the dynamic structure of the P2P network nodes can adaptively / exit, has good scalability, robustness, melon uniform distribution node has the ability to self-organization. In practice, since DHT maintenance mechanism is more complex, especially due to exit nodes frequently join the network will greatly increase the volatility of structured P2P network DHT maintenance expense; and, DHT-based resources to find support only exact match keyword matching inquiry, cannot support the content / semantic complex queries that it cannot meet the needs of a dynamic cloud environment to find a scalable resource node data management and data resources. Thus, the topology of structured P2P networks are generally used for resource management and resource lookup LAN. Structured P2P network corresponding to the unstructured P2P network. In the unstructured P2P network, each node can be arbitrarily selected node as the rest of the neighbors to establish contact, since the connection between nodes arbitrary large, the extension of the structure thus formed loose. Unstructured overlay network topology formed nearly random graph typically unstructured P2P network includes Gnutellatwi, Kazaatwi like. Unstructured topology allows the system in the management of large-scale randomized join or leave the data resource nodes, with lower maintenance costs for highly dynamic environment. And, in an unstructured P2P network, data resources can be designed and flexible search algorithms, efficient data collection resources. These characteristics unstructured P2P structure, making it possible to manage resource nodes and resources to deal with a dynamic and flexible network environment to find, and has been widely used in video and network file sharing. Typically, large build data services, often involving large scale and growing large data processing tasks that require high-capacity support scalable storage system reliability and business computing system, and the general budget of small and medium enterprises or organizations and limited financial resources, can not be equipped with the infrastructure of Gao expensive to meet the performance and scalability of large information data processing needs. And, by the enterprises to build their own infrastructure, large data processing system, it usually means expensive post-maintenance investment. Thus, in the face of large-value data it contains rich while expensive infrastructure investment and maintenance costs later make SMEs feel powerless and frustrated. Cloud computing demand access, pay-per-use, nearly unlimited expansion of resource use, provides a self-configuring resource pool play through network access (including network, virtual machine technology, storage, applications and other resources), and only a small amount of the management will be able to quickly obtain or release build dynamic computing and storage resources in the data necessary for the service. The emergence of cloud computing model, $\mathrm{W}$ and increasingly cloudy service provider for large data services Kyrgyzstan services for the realization of large low-cost data service provides a strong technical support to build. In general, the face of a wide range of big data applications and services, it is difficult to find a cloud service in full compliance with their application needs, functions as a cloud service provider cloud services are relatively 
simple, such as laaS infrastructure services, PaaS platform cloud services and SaaS software applications and services. These cloud services are usually only meet the needs of a large part of the application data services. Therefore, we need to demand the application of large data services decomposed by mission planning, the large complex data processing tasks into a single function, and mutually independent sub-tasks. By functionally independent subtasks match cloud services to meet their needs, to form a combined cloud services program to meet the large demand for data services, computing resources and storage resources.

Mission planning big data services, is based on the application requirements of large data services through manual or computer-assisted automated way to process large data processing complex calculations involved in the process of decomposition. Functional or computational complexity of big data processing task decomposition to function independently and function relatively simple sub-tasks, sub-tasks synergistic fit over each other to complete large, complex data analysis process. Processing of large data life cycle can be used to represent a combination of services, a combination of individual atoms and services corresponding to the service of large data processing tasks planned for each sub-task. Currently, based on the combined service division of tasks including automated and manual tasks into task partitioning.

\subsection{Big Data Services Trusted Combination of Assessment Techniques}

Mission planning based big data services, data services trusted combination of ideological discipline is through the combined technologies and services, according to the results of large data service mission planning, for each function independent subtasks, its Dissatisfied with Cable cloud computing resources provided cloud service (generally refers to the cloud data center, cloud platform services, or cloud software applications); then, QoS-based cloud services (ie non-functional property values) and QoS large data processing of the cloud service constraints such as budget, the failure rate and other QoS restrictions on credible candidate cloud services portfolio assessment, select the optimal combination of QoS scheme, complete the application needs of large data processing, large data services based on a combination of trusted cloud services. QoS metrics of cloud services for non-functional properties of evaluating cloud services, is used to describe the service user satisfaction with the services, usually as calculated on the basis of large data service portfolio assessment. In actual use, provide the same or similar functions of cloud services on their QoS indicators there is a big difference, each dimension QoS indicators of a different nature, affecting the value of its index of user satisfaction are also different. Specifically, the cloud service QoS values by their nature can be divided into two categories: efficiency indicators and cost index, the former value, the higher the higher the degree of user satisfaction, such as usability, availability and credibility and other indicators; the latter value the higher the user satisfaction, such as the price of services, response time and other indicators.

\section{The Proposed Scheme}

Text mining is to identify potential knowledge from unstructured text. Text data mining Digital Library is intended primarily for HTML or XML document set. This network structure is no text, or have a limited structure, therefore, first of all to such a document into the middle class as a document that reflects the characteristics of the content of representation, the general characteristics of the text representation, then using text classification and text poly and other types of machine learning methods to extract knowledge model. Text mining and data mining has a certain distinction, from the discovery of the relationship between the data, the text mining and data mining is very similar, but data mining face is structured data, the approach is mostly very clear quantitative methods. Mining and text mining because it is unstructured text, so it uses the 
method and data mining is different. Text mining and natural language understanding are often used for handling text, such as text summarization, classification, text retrieval technology. Text mining, knowledge discovery is often not accurate data, but qualitative rules. Data processing data mining is structured, wherein the amount is usually relatively small, and the text data is unstructured or semi-structured, and converted to feature vector feature data is relatively large, so the most important issue facing is how text mining in computer fair representation of text. This notation is necessary to contain sufficient information to reflect the characteristics of the text, but also not too large, so that the learning algorithm can not handle, so it relates to text feature extraction and screening.

Multimedia Feature extraction is the basic premise of mining. Multimedia data mining and query needs to be stored separately in the feature extraction and feature database. During the retrieval process, the search subsystem is characterized in the data rather than the database project itself, and therefore, the quality of extracted features determines retrieval effectiveness. If a feature is not extracted from the data items, then the corresponding feature query to retrieve the data it is impossible to project. In a traditional database management system, all properties are given and are complete, and in the multimedia database management system, the feature is based on the desired type of query and extraction, and usually incomplete. Object-oriented database model features and data types, including the content and characteristics of the objective characteristics of the pretreatment automatically extract user input, such as color, texture, shape, and keywords, metadata, etc. In addition, many also stores descriptive features, such as image file name, image type, a group known to the image-related keywords. Characterized by a set of vector set, he said each vector has a corresponding visual features, such as color, direction, color and layout border layout. Therefore, in general, there are three levels of multimedia data object characteristics: metadata, text annotation, content features.

Cloud computing environment, the construction of digital libraries include computing resources, digital information resources, storage space, user interface and application information systems, according to the characteristics of cloud computing technology at present, application status and associated data technology to build digital library actual demand conditions. Application platform is the top layer of the entire digital library of cloud services platform, is the core part of the library of cloud services platform, which includes software layer and the platform layer. Among them, the software layer integrates a variety of library applications, business management systems, and a variety of Web server set, a library core business application platform.

Platform layer mainly contains various API interfaces, API interface is divided into public and private API interface libraries can achieve docking variety of Web applications through an interface on the digital library to provide the necessary platform for the cloud service library users features and services, including user management system library, virtual reference systems, electronic resource management systems, knowledge management systems and information retrieval system, which provides users with a variety of convenient software application services and personalized UI interface. This layer provides the user login Digital Library web portal cloud service platform, authorized users can connect to the internet based on access keys visit the Web site, where the user authorization can be authorized user on demand cloud platform hire some or all of the service, authorized by the user is able to sign a unified interface and operating platform to achieve the desired services, including interlibrary loans, on behalf of behalf of the prosecution check, online reference service, knowledge service, cloud ILS and the like. Wherein the digital library maintenance team unified cloud services platform to platform architecture maintained by the deployment of the control layer, management, coordination, handling a variety of issues at all levels of abnormal appearing, and cloud services platform in hardware and software, electronic information resources, database maintenance, update, upgrade, periodic inspection and finishing. Data layer, connecting bridge infrastructure and software application platform layer between the layers, which is 
mainly responsible for the unified management of heterogeneous database layer of data, including data management, scheduling, data fusion, data deduplication, distribution, data backup and restore , compression, data communications, data encryption and data security data. The use of Web middleware layer for heterogeneous databases on the library cloud service platform to virtualize different nodes, so long as the library integrates cloud middleware platform library node, you can easily expand data sharing network. At the same time, the use of point metadata harvesting technology on heterogeneous database node, to improve access speed and parallel processing efficiency Pavilion cloud service platform database.

\subsection{Mobile Library Cloud Platform}

Cloud data processing platform is the core of the system of data storage and data processing platform, the platform structure to a distributed file system (HDFS) and parallel computing framework (MapReduce), based integration interfaces to external systems, multi-dimensional analysis of massive data knowledge mining, data The results show a variety of services, as shown in Figure 1, the system can support large-scale distributed data acquisition, multi-dimensional analysis of parallel and parallel data mining.

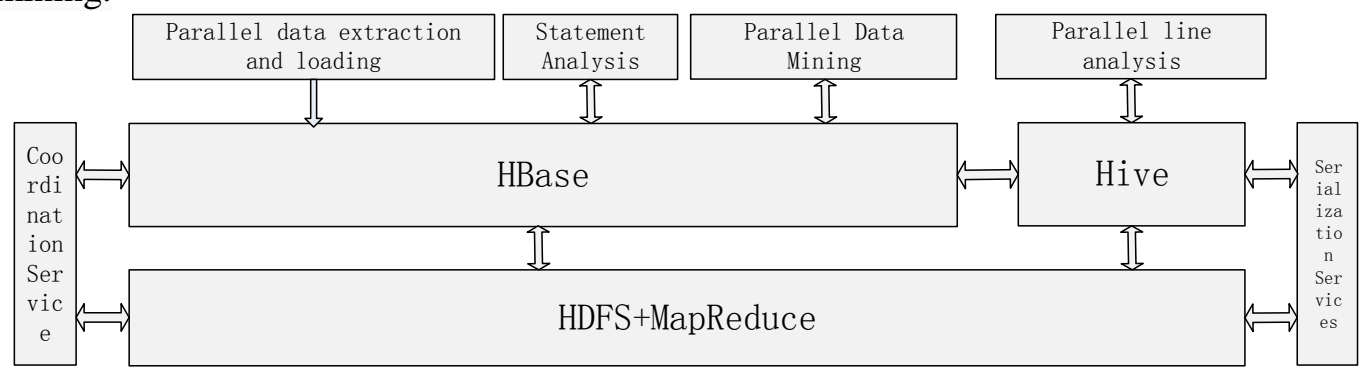

Figure 1. Mobile Library Cloud Platform

As can be seen from the figure, the main provider of cloud data processing platform features four categories, which are distributed data storage, parallel data extraction, transformation and loading (ie, data ETL), parallel and parallel data mining multi-dimensional analysis. Wherein the distributed data storage uses HBase to store data, which can greatly improve the efficiency of data access, since the underlying storage HBase uses HDFS, so that a distributed data storage, safe and reliable. ETL data used to complete the data source through the extraction, transformation, loading process into a series of HBase. Parallel to achieve multi-dimensional analysis using Hive, class Hive SQL interface provides easy to use, multi-dimensional analysis greatly simplifies the difficulty of implementation. MapReduce parallel data mining based on parallel computing framework to achieve clustering, classification, prediction, outlier analysis and other data mining algorithms make it possible to discover valuable knowledge from massive data.

ETL is a data extraction (Extract), conversion (Transform), Cleaning (Cleansing), loading (Load) process in this system is mainly used in the marketing business, weather, and other data is loaded into the information collection to the cloud platform. In order to improve the extraction, transformation and loading speed related data, you can use cloud computing to achieve parallelism of the ETL and incremental process. Based on the calculation shown in FIG ETL processes MapReduce framework follows from the figure can be seen, parallel to the first data source ETL technology reasonable block, then assign each piece of data to multiple different nodes of Map / Reduce simultaneously ETL, achieve parallelization of the process, thereby improving the efficiency of the ETL. At the same time, the process of realization of the incremental ETL process to ensure that the actual application process ETL continuity. As Figure 2 


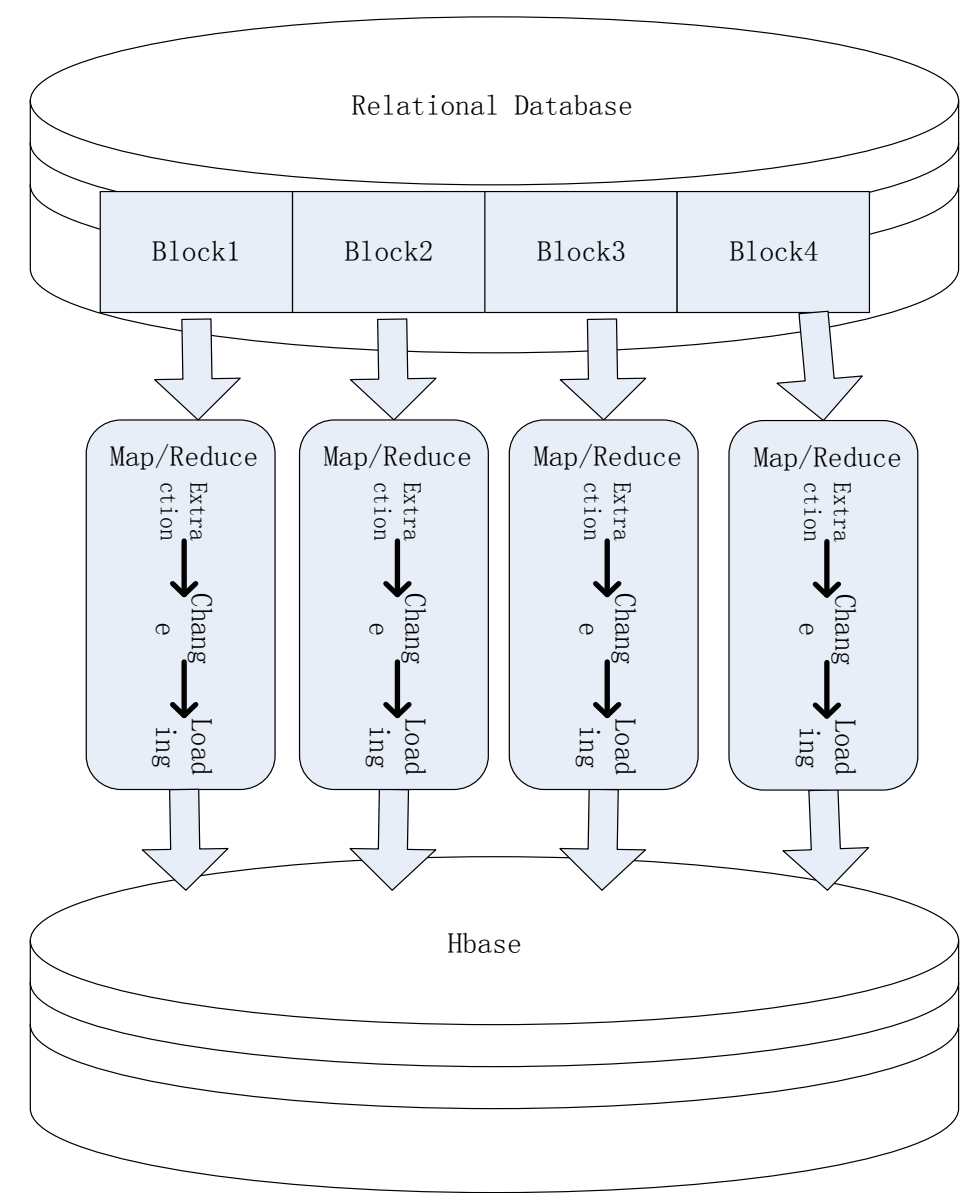

Figure 2. Map Reduce Computation Flowchart based ETL Framework

\subsection{Big Data Mining}

Based on the principle of random sparse matrix after analysis of large data compression to accelerate the convergence of the algorithm, and the algorithm also avoids a lot of scheduling data and a lot of storage. At each sampling time, the sampling of the data, the data is represented as a vector, this vector can be expressed as $\mathbf{x}_{i}$, and $\mathbf{x}_{i} \in R^{N}$, For the number of data samples referred to as $T$, and in fact, a huge number of numbers. And these huge sample data collection form a function, this function Features Big Data is having a heterogeneous and diverse characteristics, and in fact, a huge number of numbers. And these huge sample data collection form a function $f\left(\mathbf{x}_{1}, \mathbf{x}_{2}, \cdots, \mathbf{x}_{T}\right)$, this function Features Big Data is having a heterogeneous and diverse characteristics, Features Big Data is having a heterogeneous and diverse characteristics of the variables or parameters described as a large, complex system or subsystem. As for data acquisition $\mathbf{x}_{1}, \mathbf{x}_{2}, \cdots, \mathbf{x}_{T}$ represents a dimension of the sample, As for the dimension $N$ data representing the samples collected, the data matrix large orders $\mathbf{X} \in \square^{N \times T}$, when one of the elements to meet their zero mean and variance; if the variance $\sigma^{2}$ is a finite number, and where the elements meet the characteristics of independent and identically distributed, according to the independent and identically distributed the characteristics of the research point of a special class of random matrix Euclid, having

$$
\mathbf{M}_{n}=\left(f_{n}\left(\left\|x_{i}-x_{j}\right\|^{2}\right)\right)_{n \times n}
$$

Consider a Gaussian random matrix Euclid, according to the theory of random matrices, 
the upper bound of the spectrum of this measure is

$$
d\left(F^{\mathbf{x}_{1}}, F^{\mathbf{x}_{2}}\right) \leq \sqrt{\frac{1}{T} \operatorname{tr}\left(\mathbf{X}_{1}-\mathbf{X}_{2}\right)} \wedge \frac{\operatorname{rank}\left(\mathbf{X}_{1}-\mathbf{X}_{2}\right)}{T}
$$

As for the similarity determination data, we need to find similarity metrics of two vectors, in the present study, the distance between the spectral measure, referred to as

$$
d\left(F^{\mathbf{x}_{1}}, F^{\mathbf{x}_{2}}\right)
$$

\section{Conclusion}

Based on the characteristics of cloud mobile library service, to build large-scale data mining method based on random matrix of large data mining, this method can quickly retrieve the relevant data, and search and recommendations based on the relevant data, and based on this data mining method It proposed to build a distributed computing network cloud. This method can be used in mobile applications under the mobile Internet.

\section{References}

[1] Beenakker C W J. Random-matrix theory of Majorana fermions and topological superconductors[J]. Reviews of Modern Physics, 2015, 87(3): 1037.

[2] Majumdar S N, Schehr G. Top eigenvalue of a random matrix: large deviations and third order phase transition[J]. Journal of Statistical Mechanics: Theory and Experiment, 2014, 2014(1): P01012.

[3] Shahjahan S, Aubry A, Rupin F, et al. A random matrix approach to detect defects in a strongly scattering polycrystal: How the memory effect can help overcome multiple scattering[J]. Applied Physics Letters, 2014, 104(23): 234105.

[4] Entin A, Roditty-Gershon E, Rudnick Z. Low-lying zeros of quadratic Dirichlet L-functions, hyper-elliptic curves and Random Matrix Theory [J]. Geometric and Functional Analysis, 2013, 23(4): 1230-1261.

[5] Erdős L, Yau H T, Yin J. Rigidity of eigenvalues of generalized Wigner matrices[J]. Advances in Mathematics, 2012, 229(3): 1435-1515.

[6] Nagata K, Nishimura J, Shimasaki S. Gauge cooling for the singular-drift problem in the complex Langevin method--a test in Random Matrix Theory for finite density $\mathrm{QCD}[\mathrm{J}]$. arXiv preprint arXiv:1604.07717, 2016.

[7] Dubbs A, Edelman A. Infinite random matrix theory, tridiagonal bordered Toeplitz matrices, and the moment problem[J]. Linear Algebra and its Applications, 2015, 467: 188-201.

[8] El Alaoui M. Random matrix theory and portfolio optimization in Moroccan stock exchange[J]. Physica A: Statistical Mechanics and its Applications, 2015, 433: 92-99.

[9] Shahjahan S, Aubry A, Rupin F, et al. Improvement of flaw detection with ultrasonic array probes in multiple scattering polycrystalline materials by means of a random matrix approach: experimental and simulation studies[J]. NDCM-XIII, Le Mans, France, 20-24 May, 2013.

[10] Potters M. A Random Matrix Bayesian framework for out-of-sample quadratic optimization[J]. 2013.

\section{Authors}

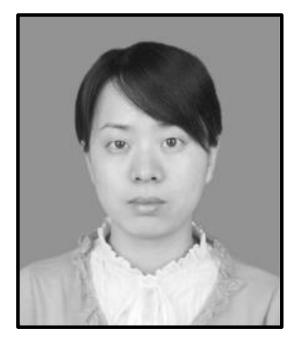

Jing $\mathbf{L i}$ received the M.S. degree in Computer application and technology from Inner Mongolia normal university in 2008. She is a lecturer at Zhoukou Normal University. Her research interests include service computing, image processing, etc.

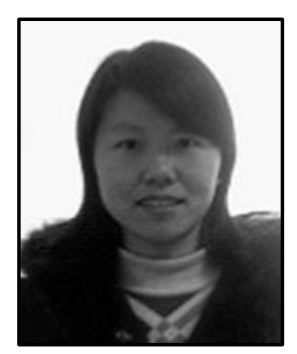

Chunying Cui, She received the M.S. degree in Computer application and technology from Huazhong University of Science and Technology in 2007. She is a Associate professor at Zhoukou Normal University. Her research interests include data security, etc. 